\title{
Transmuted Mukherjee-Islam Distribution: A Generalization of Mukherjee-Islam Distribution
}

\author{
Loai M. A. Al-Zou'bi ${ }^{1}$ \\ ${ }^{1}$ Al al-Bayt University, Department of Mathematics, Mafraq (25113), Jordan \\ Correspondence: Al al-Bayt University, Department of Mathematics, Mafraq (25113), Jordan. E-mail: loai67@ yahoo.com
}

Received: April 26, 2017 Accepted: June 28, 2017 Online Published: July 24, 2017

doi:10.5539/jmr.v9n4p135 URL: https://doi.org/10.5539/jmr.v9n4p135

\begin{abstract}
A new continuous distribution is proposed in this paper. This distribution is a generalization of Mukherjee-Islam distribution using the quadratic rank transmutation map. It is called transmuted Mukherjee-Islam distribution (TMID). We have studied many properties of the new distribution: Reliability and hazard rate functions. The descriptive statistics: mean, variance, skewness, kurtosis are also studied. Maximum likelihood method is used to estimate the distribution parameters. Order statistics and Renyi and Tsallis entropies were also calculated. Furthermore, the quantile function and the median are calculated.
\end{abstract}

Keywords: Transmuted Mukherjee-Islam distribution, Moments, Entropy, Order statistics, quantile function

\section{Introduction}

Shaw and Buckley (2007) have proposed to transmutation maps, the sample and rank transmutations. The simplest rank transmutation map is the quadratic rank transmutation map. The quadratic rank transmutation map will be used through this paper to derive a generalization of the Mukherjee-Islam distribution with some of its properties. This generalization is called the transmuted Mukherjee-Islam (TMI) distribution. Al-Omari et al. (2017) proposed the transmuted janadran distribution as a generalization of the Janadran distribution. Aryal and Tsokos (2011) worked out a generalization of the weibull probability distribution (transmuted weibull distribution). Merovci (2013a) used the quadratic rank transmutation map to develop a new distribution called the Transmuted Lindley Distribution. Merovci (2013b) used this map to develop a Transmuted Rayleigh Distribution. An extension of the exponentiated generalized $G$ class of distributions (Cordeiro et al., 2013) called the transmuted exponentiated generalized $G$ family. A simple representation for the transmuted $G$ family density function as a linear mixture of the $G$ and the exponentiated- $G$ densities was derived by Bourguignon et al. (2016). Many authors worked out generalizations to some distributions using the quadratic rank transmutation map. For example Merovci and Elbatal (2014) introduced the Transmuted Lindley-Geometric distribution, whereas, Vardhan and Balaswamy (2016) proposed a transmuted new modified Weibull distribution. A Transmuted Lomax distribution (Ashour and Eltehiwy, 2013), a Transmuted Log-Logistic Distribution (Aryal and Tsokos, 2013), Transmuted Burr Type XII Distribution (Khazaleh, 2016). El- batal et al. (2014) studied some general properties of the transmuted exponentiated Frêchet distribution. Based on new modified weibull distribution, Vardhan and Balaswamy (2016) produced a transmuted distribution using the quadratic rank transmutation map, named transmuted new modified weibull distribution. A transmuted modified weibull distribution is introduced by Khan and King (2013).

We organized the rest of this paper as follows: In Section 2 the pdf and CDF of the TMI distribution are demonstrated. In Section 3, the reliability and hazard rate functions of our model are computed. We summarized the distributions of order statistics in Section 4. Some properties, like the $r^{\text {th }}$ moment, mean, variance, skewness, kurtosis, coefficient of variation and the moment generating function of the TMI distribution are derived in Section 5. In Section 6 the maximum likelihood estimates of the distribution parameters are demonstrated. The Renyi and Tsallis entropies are calculated in Section 7. The quantile function is derived in Section 8. Finally, in Section 9 we will draw conclusions.

\section{Transmuted Mukherjee-Islam Distribution}

A random variable, $X$, is said to have a Mukherjee-Islam distribution (Mukheerji and Islam, 1983) with parameters $\theta$ and $p$ if it has a cumulative distribution function $(C D F)$

$$
W(x)=\left\{\begin{array}{cc}
0, & x<0 \\
\frac{x^{p}}{\theta^{p}}, & 0<x \leq \theta \\
1, & x>\theta
\end{array}\right.
$$


with a corresponding probability density function $(p d f)$ given by:

$$
w(x)=\left\{\begin{array}{lc}
\frac{p x^{p-1}}{\theta^{p}}, & 0<x \leq \theta, \theta, p>0 \\
0, & \text { otherwise }
\end{array}\right.
$$

Definition 2.1 A random variable $X$ is said to have a transmuted distribution if its $C D F$ is given by

$$
\Psi(x)=(1+\lambda) W(x)-\lambda[W(x)]^{2}, \quad|\lambda| \leq 1
$$

where $W(x)$ is the $C D F$ of the base distribution. The $p d f$ of the transmuted random variable is given by

$$
\psi(x)=w(x)(1+\lambda-2 \lambda W(x))
$$

The CDF of this random variable is, hence, defined using Equations (1) and (3) as:

$$
\Psi(x)=(1+\lambda) \frac{x^{p}}{\theta^{p}}-\lambda \frac{x^{2 p}}{\theta^{2 p}}, 0<x \leq \theta
$$

Therefore, the pdf of the transmuted Mukherjee-Islam random variable, X, is defined using Equations (1), (2) and (4) as:
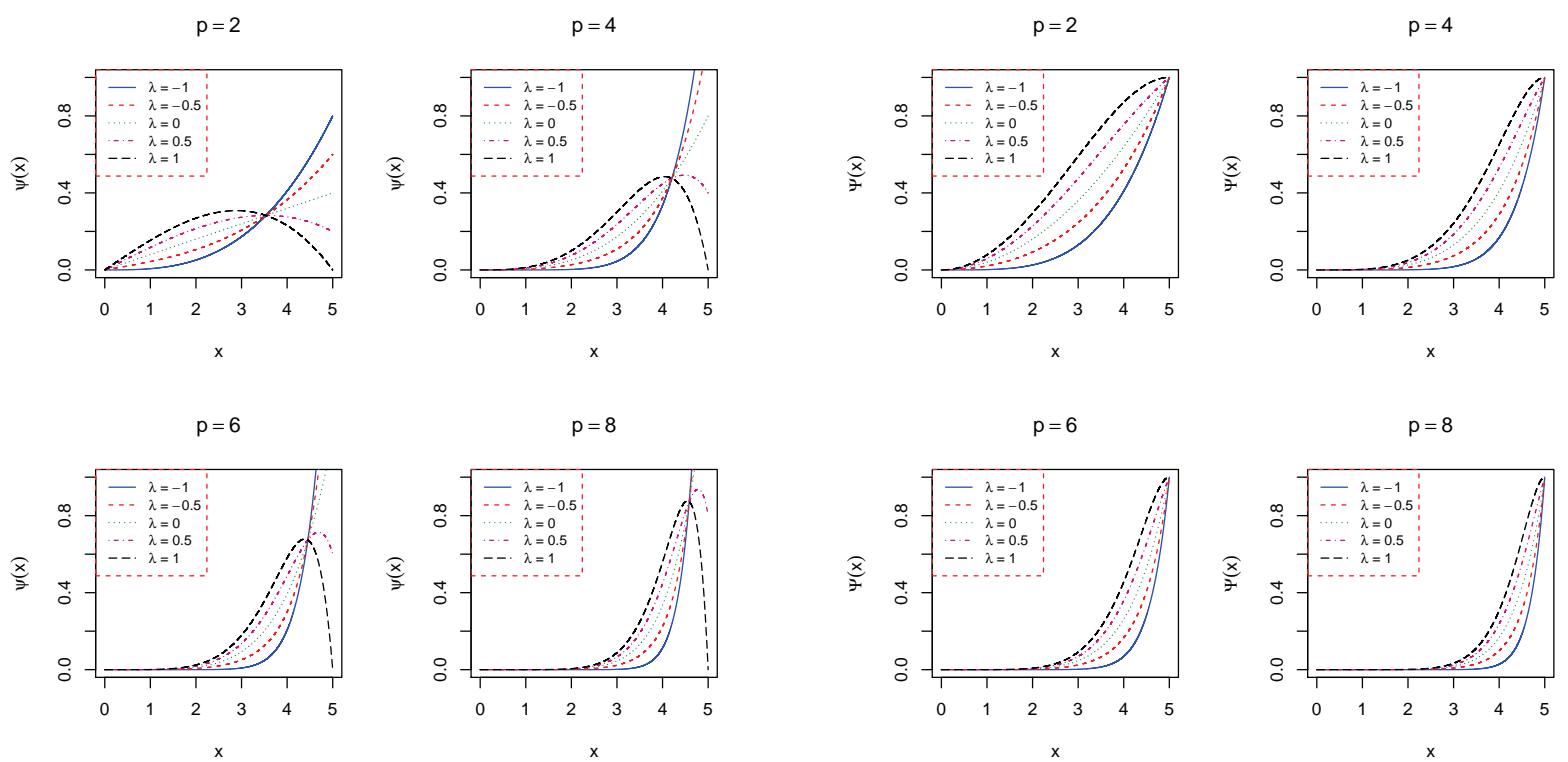

(a) The pdf of the TMI distribution with different values of (b)The CDF of the TMI distribution with different values of $p, \lambda$ when $\theta=5$

$p, \lambda$ when $\theta=5$

Figure (1a) shows the pdf of the TMI for $\theta=5$ and different values of $p, 2,4,6$ and 8 . We varied the value of $\lambda$ from -1 to 1 with a step of 0.5 . The figure shows that the TMI random variable has a left skewed distribution. The tail of the distribution gets heavier as the value of $\lambda$ gets smaller. Figure (1b) shows the plot of the CDF of the TMI random variable for $\theta=5$ with $p$ equals to $2,4,6$ and 8 and $\lambda=-1,-0.5,0,0.5$, and 1 .

\section{Reliability Analysis}

The reliability and hazard rate functions are defined by:

$$
\begin{aligned}
& R(t)=1-\Psi(t) \\
& H(t)=\frac{\psi(t)}{1-\Psi(t)}
\end{aligned}
$$


Theorem 3.1 The reliability and hazard rate functions of the TMI distribution, respectively are

$$
\begin{aligned}
R(t) & =1-\frac{t^{p}}{\theta^{p}}\left[1+\lambda-\lambda \frac{t^{p}}{\theta^{p}}\right] \\
H(t) & =\frac{p t^{p-1}\left[(1+\lambda) \theta^{p}-2 \lambda t^{p}\right]}{\theta^{2 p}-t^{p}\left[(1+\lambda) \theta^{p}-\lambda t^{p}\right]}
\end{aligned}
$$

Proof. The proof of the reliability is straightforward, by substituting the $C D F$ of the TMI distribution Equation (5) in Equation (7). Now, for the hazard rate function, substituting Equations (5) and (6) in Equation (8), we get

$$
\begin{array}{r}
H(t)=\frac{\frac{(1+\lambda) p}{\theta^{p}} t^{p-1}-\frac{2 \lambda p}{\theta^{2 p}} t^{2 p-1}}{1-\frac{t^{p}}{\theta^{p}}\left[1+\lambda-\lambda \frac{t^{p}}{\theta^{p}}\right]} \\
=\frac{p t^{p-1}\left[(1+\lambda) \theta^{p}-2 \lambda t^{p}\right]}{\theta^{2 p}-t^{p}\left[(1+\lambda) \theta^{p}-\lambda t^{p}\right]}
\end{array}
$$
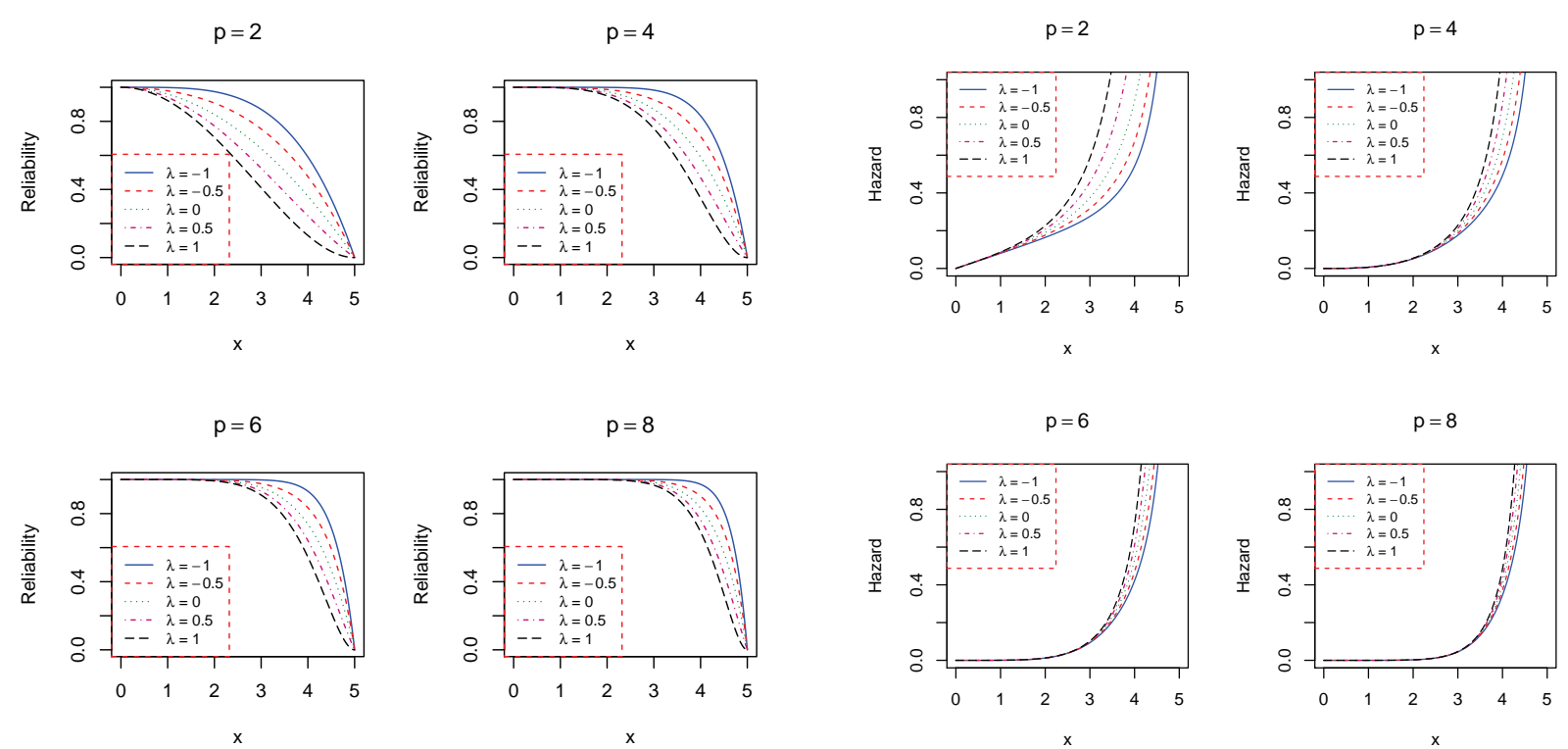

(a) Reliability of the TMID with different values of $p, \lambda$ when $\theta=5$
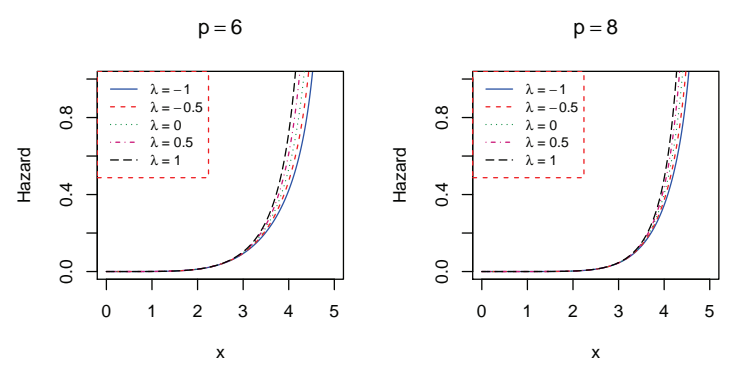

(b) Hazard rate function of the TMID with different values of $p, \lambda$ when $\theta=5$

\section{Order Statistics}

Let $X_{1}, X_{2}, \ldots X_{n}$ be a random sample with $p d f \psi(x)$ and $C D F \Psi(x)$. If $X_{(1)}, X_{(2)}, \ldots X_{(n)}$ is the order statistic of this sample, where $X_{(1)} \leq X_{(2)} \leq \ldots \leq X_{(n)}$. Then the $p d f$ of the $j^{\text {th }}$ order statistics, $X_{(j)}$ is given by:

$$
\psi_{(j)}(x)=j\left(\begin{array}{l}
n \\
j
\end{array}\right) \psi(x)[\Psi(x)]^{j-1}[1-\Psi(x)]^{n-j},
$$

Substituting $j=1$ in Equation (9), we get the $p d f$ of first order statistics $X_{(1)}=\min \left(X_{1}, X_{2}, \ldots X_{n}\right)$.

$$
\psi_{(1)}(x)=n p \frac{x^{p}}{\theta^{p}}\left(1+\lambda-2 \lambda \frac{x^{p}}{\theta^{p}}\right)\left(1-(1+\lambda) \frac{x^{p}}{\theta^{p}}+\lambda \frac{x^{2 p}}{\theta^{2 p}}\right)^{(n-1)}, \quad x \in(0, \theta)
$$


The $p d f$ of the $n^{\text {th }}$ order statistic $X_{(n)}=\max \left(X_{1}, X_{2}, \ldots X_{n}\right)$, is defined as:

$$
\psi_{(n)}(x)=n p \frac{x^{p}}{\theta^{p}}\left(1+\lambda-2 \lambda \frac{x^{p}}{\theta^{p}}\right)\left((1+\lambda) \frac{x^{p}}{\theta^{p}}-\lambda \frac{x^{2 p}}{\theta^{2 p}}\right)^{(n-1)}
$$

Furthermore, for any value of $j$ the common form of $\psi_{(j)}(x)$ can be obtained as

$$
\begin{aligned}
\psi_{(j)}(x) & =j\left(\begin{array}{l}
n \\
j
\end{array}\right)\left[\frac{(1+\lambda) p}{\theta^{p}} x^{p-1}-\frac{2 \lambda p}{\theta^{2 p}} x^{2 p-1}\right]\left[(1+\lambda) \frac{x^{p}}{\theta^{p}}-\lambda \frac{x^{2 p}}{\theta^{2 p}}\right]^{j-1} \\
& \times\left[1-(1+\lambda) \frac{x^{p}}{\theta^{p}}+\lambda \frac{x^{2 p}}{\theta^{2 p}}\right]^{n-j} \\
& =j\left(\begin{array}{l}
n \\
j
\end{array}\right) \frac{x^{p j-1}}{\theta^{p j}}\left[(1+\lambda) p-2 \lambda p \frac{x^{p}}{\theta^{p}}\right]\left[1+\lambda-\lambda \frac{x^{p}}{\theta^{p}}\right]^{j-1}\left[1-(1+\lambda) \frac{x^{p}}{\theta^{p}}+\lambda \frac{x^{2 p}}{\theta^{2 p}}\right]^{n-j}
\end{aligned}
$$

\section{Moments}

\section{$5.1 r^{\text {th }}$ Moment}

Theorem 5.1 The $r^{\text {th }}$ moment of the TMI random variable is defined as:

$$
E\left(X^{r}\right)=\left(\frac{2 p^{2}+r p-r p \lambda}{(r+p)(r+2 p)}\right) \theta^{r}
$$

Proof.

$$
\begin{aligned}
E\left(X^{r}\right) & =\int_{0}^{\theta} x^{r} \psi(x) d x \\
& =\int_{0}^{\theta} x^{r}\left(\frac{(1+\lambda) p}{\theta^{p}} x^{p-1}-\frac{2 \lambda p}{\theta^{2 p}} x^{2 p-1}\right) d x \\
& =\frac{p(1+\lambda)}{\theta^{p}} \int_{0}^{\theta} x^{r+p-1} d x-\frac{2 p \lambda}{\theta^{2 p}} \int_{0}^{\theta} x^{r+2 p-1} d x \\
& =\frac{p(1+\lambda)}{\theta^{p}}\left(\frac{\theta^{r+p}}{r+p}\right)-\frac{2 p \lambda}{\theta^{2 p}}\left(\frac{\theta^{r+2 p}}{r+2 p}\right) \\
& =\left(\frac{2 p^{2}+r p-r p \lambda}{(r+p)(r+2 p)}\right) \theta^{r}
\end{aligned}
$$

5.1.1 Mean, Variance, Skewness, Kurtosis and Coefficient of Variation

The first and the second moments can be computed by replacing $r$ by 1 and 2; respectively in (13) as follows:

$$
\begin{aligned}
\mu=E(X) & =\left(\frac{2 p^{2}+p-p \lambda}{(1+p)(1+2 p)}\right) \theta \\
E\left(X^{2}\right) & =\left(\frac{p^{2}+p-p \lambda}{(2+p)(1+p)}\right) \theta^{2}
\end{aligned}
$$

But the variance of a random variable is defined as $\operatorname{var}(X)=E\left(X^{2}\right)-(E(X))^{2}$, therefore

$$
\begin{aligned}
\operatorname{var}(X) & =\left(\frac{p^{2}+p-p \lambda}{(2+p)(1+p)}\right) \theta^{2}-\left(\frac{2 p^{2}+p-p \lambda}{(1+p)(1+2 p)}\right)^{2} \theta^{2} \\
& =\frac{-p\left(p^{2} \lambda^{2}+2 p \lambda^{2}-2 p^{2} \lambda+p \lambda+\lambda-4 p^{2}-4 p-1\right)}{(1+p)^{2}(2+p)(1+2 p)^{2}} \theta^{2}
\end{aligned}
$$


The coefficient of variation $(\mathrm{cv})$ is defined to be the ratio of standard deviation of the random variable to it expected value, that is $c v=\frac{\sqrt{\operatorname{var}(X)}}{E(X)}$. Therefore,

$$
c v=\frac{\sqrt{-p\left(p^{2} \lambda^{2}+2 p \lambda^{2}-2 p^{2} \lambda+p \lambda+\lambda-4 p^{2}-4 p-1\right)}}{\left(2 p^{2}+p-p \lambda\right) \sqrt{2+p}}
$$

The third and fourth moments of the random variable $X$ can be determined by replacing $r$ by 3 and 4; respectively in Equation (13). Thus, they are given by:

$$
\begin{aligned}
& E\left(X^{3}\right)=\left(\frac{2 p^{2}+3 p-3 p \lambda}{(3+p)(3+2 p)}\right) \theta^{3} \\
& E\left(X^{4}\right)=\left(\frac{2 p^{2}+4 p-4 p \lambda}{(4+p)(4+2 p)}\right) \theta^{4}
\end{aligned}
$$

The skewness and the kurtosis of a random variable are defined as:

$$
\begin{aligned}
s k(X) & =\frac{E\left(X^{3}\right)-3 E(X) \operatorname{var}(X)-(E(X))^{3}}{(\operatorname{var}(X))^{\frac{3}{2}}} \\
k \operatorname{kur}(X) & =\frac{E\left(X^{4}\right)-4(E(X))\left(E\left(X^{3}\right)\right)+6(E(X))^{2} \operatorname{var}(X)+3(E(X))^{4}}{(\operatorname{var}(X))^{2}}
\end{aligned}
$$

Based on the first four moments, the skewness and the kurtosis of the TMI random variable, $X$ are given by:

$$
\begin{aligned}
S k(X)= & \frac{\left(2 p^{2}+3 p-3 p \lambda\right)\left((1+p)^{3}(1+2 p)^{3}(2+p)^{\frac{3}{2}}\right)}{(3+p)(3+2 p)\left(-p\left(p^{2} \lambda^{2}+2 p \lambda^{2}-2 p^{2} \lambda+p \lambda+\lambda-4 p^{2}-4 p-1\right)\right)^{\frac{3}{2}}} \\
- & \frac{\left(2 p^{2}+p-p \lambda\right)^{3}(2+p)^{\frac{3}{2}}}{\left(-p\left(p^{2} \lambda^{2}+2 p \lambda^{2}-2 p^{2} \lambda+p \lambda+\lambda-4 p^{2}-4 p-1\right)\right)^{\frac{3}{2}}} \\
- & \frac{3\left(2 p^{2}+p-p \lambda\right) \sqrt{(2+p)}}{\sqrt{\left(-p\left(p^{2} \lambda^{2}+2 p \lambda^{2}-2 p^{2} \lambda+p \lambda+\lambda-4 p^{2}-4 p-1\right)\right)}} \\
\operatorname{kur}(X) & =\frac{\left(p^{2}+2 p-2 p \lambda\right)(1+p)^{4}(2+p)(1+2 p)^{4}}{(2+p)\left(-p\left(p^{2} \lambda^{2}+2 p \lambda^{2}-2 p^{2} \lambda+p \lambda+\lambda-4 p^{2}-4 p-1\right)\right)} \\
& -\frac{4(1+p)^{3}(2+p)^{2}(1+2 p)^{3}(2 p+1-\lambda)(2 p+3-3 \lambda)}{(3+p)(3+2 p)\left(p^{2} \lambda^{2}+2 p \lambda^{2}-2 p^{2} \lambda+p \lambda+\lambda-4 p^{2}-4 p-1\right)} \\
& +\frac{[(1+p)(2+p)(1+2 p)(2 p+1-\lambda)]^{2}}{p^{2} \lambda^{2}+2 p \lambda^{2}-2 p^{2} \lambda+p \lambda+\lambda-4 p^{2}-4 p-1} \\
& +\frac{4 p^{2}(2 p+1-\lambda)(2+p)^{2}}{\left(p^{2} \lambda^{2}+2 p \lambda^{2}-2 p^{2} \lambda+p \lambda+\lambda-4 p^{2}-4 p-1\right)^{2}}
\end{aligned}
$$


Table 1. The mean, standard deviation, skewness, kurtosis and the coefficient of variation of the TMI distribution for different values of $\lambda$ when $p=2$ and $\theta=5$

\begin{tabular}{c|ccccc}
\hline$\lambda$ & $\mu=E\left(X_{T M I}\right)$ & $\sigma_{X}$ & $S k(X)$ & $\operatorname{Kur}(X)$ & $C V(\%)$ \\
\hline-1.0 & 4.000 & 0.816 & -1.050 & 3.696 & 20.412 \\
-0.9 & 3.933 & 0.883 & -1.103 & 3.883 & 22.438 \\
-0.8 & 3.867 & 0.939 & -1.088 & 3.776 & 24.291 \\
-0.7 & 3.800 & 0.988 & -1.042 & 3.574 & 26.007 \\
-0.6 & 3.733 & 1.031 & -0.981 & 3.352 & 27.606 \\
-0.5 & 3.667 & 1.067 & -0.913 & 3.138 & 29.105 \\
-0.4 & 3.600 & 1.098 & -0.842 & 2.944 & 30.513 \\
-0.3 & 3.533 & 1.125 & -0.771 & 2.774 & 31.839 \\
-0.2 & 3.467 & 1.147 & -0.701 & 2.627 & 33.086 \\
-0.1 & 3.400 & 1.165 & -0.632 & 2.503 & 34.258 \\
0.0 & 3.333 & 1.179 & -0.566 & 2.400 & 35.356 \\
0.1 & 3.267 & 1.188 & -0.501 & 2.317 & 36.378 \\
0.2 & 3.200 & 1.194 & -0.440 & 2.252 & 37.326 \\
0.3 & 3.133 & 1.197 & -0.381 & 2.204 & 38.194 \\
0.4 & 3.067 & 1.195 & -0.326 & 2.172 & 38.979 \\
0.5 & 3.000 & 1.190 & -0.275 & 2.153 & 39.675 \\
0.6 & 2.933 & 1.181 & -0.230 & 2.148 & 40.276 \\
0.7 & 2.867 & 1.169 & -0.189 & 2.150 & 40.764 \\
0.8 & 2.800 & 1.152 & -0.156 & 2.161 & 41.136 \\
0.9 & 2.733 & 1.131 & -0.134 & 2.173 & 41.374 \\
1.0 & 2.667 & 1.106 & -0.125 & 2.180 & 41.457 \\
\hline
\end{tabular}

Table 1 shows the values of the mean, standard deviation, skewness, kurtosis and the coefficient of variation $(C V)$ of the TMI random variable for different values of $\lambda$ when $p=2$ and $\theta=5$. The table shows that as $\lambda$ increases the mean decreases. The kurtosis decreases as well. It, also tells us that the shape of the distribution is always skewed to the left regardless the value of $\lambda$. The shape of the distribution has sharper peak as $\lambda$ decreases. The table, as well shows that the mean of the MI distribution, which equals to 3.333, is not far from the mean of the TMI distribution for $-0.5 \leq \lambda \leq 0.5$. Both means are equal when $\lambda=0$.

\subsection{Moment Generating Function}

Theorem 5.2 The moment generating function $(M G F)$ of the TMI random variable is given by

$$
M_{x}(t)=\sum_{k=0}^{\infty} \frac{p(2 p+k-\lambda k)}{(k+p)(k+2 p)} \frac{(t \theta)^{k}}{k !}
$$

Proof.

$$
\begin{aligned}
M_{x}(t) & =E\left(e^{t x}\right) \\
& =\int_{0}^{\theta} e^{t x} \psi(x) d x \\
& =\int_{0}^{\theta}\left(\frac{(1+\lambda) p}{\theta^{p}} x^{p-1}-\frac{2 \lambda p}{\theta^{2 p}} x^{2 p-1}\right) e^{t x} d x \\
& =\frac{(1+\lambda) p}{\theta^{p}} \int_{k=0}^{\theta} x^{p-1} e^{t x} d x-\frac{2 \lambda p}{\theta^{2 p}} \int_{k=0}^{\theta} x^{2 p-1} e^{t x} d x \\
& =\frac{(1+\lambda) p}{\theta^{p}} \int_{k=0}^{\theta} x^{p-1} \sum_{k=0}^{\infty} \frac{t^{k} x^{k}}{k !} d x-\frac{2 \lambda p}{\theta^{2 p}} \int_{k=0}^{\theta} x^{2 p-1} \sum_{k=0}^{\infty} \frac{t^{k} x^{k}}{k !} d x \\
& =\frac{(1+\lambda) p}{\theta^{p}} \sum_{k=0}^{\infty} \frac{t^{k}}{k !} \int_{k=0}^{\theta} x^{k+p-1} d x-\frac{2 \lambda p}{\theta^{2 p}} \sum_{k=0}^{\infty} \frac{t^{k}}{k !} \int_{k=0}^{\theta} x^{k+2 p-1} d x \\
& =\frac{(1+\lambda) p}{\theta^{p}} \sum_{k=0}^{\infty} \frac{t^{k}}{k !(k+p)} \theta^{k+p} d x-\frac{2 \lambda p}{\theta^{2 p}} \sum_{k=0}^{\infty} \frac{t^{k}}{k !(k+2 p)} \theta^{k+2 p} d x \\
& =(1+\lambda) p \sum_{k=0}^{\infty} \frac{t^{k}}{k !(k+p)} \theta^{k} d x-2 \lambda p \sum_{k=0}^{\infty} \frac{t^{k}}{k !(k+2 p)} \theta^{k} d x \\
& =\sum_{k=0}^{\infty}\left[\frac{(1+\lambda) p}{k+p}-\frac{2 \lambda p}{k+2 p}\right] \frac{(t \theta)^{k}}{k !} \\
& =\sum_{k=0}^{\infty}\left[\frac{(1+\lambda)(k+2 p) p-2 \lambda p(k+p)}{(k+p)(k+2 p)}\right] \frac{(t \theta)^{k}}{k !} \\
& =\sum_{k=0}^{\infty} \frac{p(2 p+k-\lambda k)}{(k+p)(k+2 p)} \frac{(t \theta)^{k}}{k !}
\end{aligned}
$$




\section{Maximum Likelihood Estimates}

Definition 6.1 Let $X_{1}, X_{2}, \ldots, X_{n}$ be a random sample size $n$ with a $p d f \psi(x)$. The likelihood function is defined as the joint density of the random sample, which is defined as

$$
\ell=L\left(\lambda, \theta, p \mid x_{1}, x_{2}, \ldots, x_{n}\right)=\prod_{i=1}^{n} \psi\left(x_{i} \mid \lambda, \theta, p\right)
$$

Hence, the likelihood function is given by

$$
\begin{aligned}
\ell & =\prod_{i=1}^{n}\left(\frac{(1+\lambda) p}{\theta^{p}} x_{i}^{p-1}-\frac{2 \lambda p}{\theta^{2 p}} x_{i}^{2 p-1}\right) \\
& =\prod_{i=1}^{n} p \frac{x_{i}^{p-1}}{\theta^{p}}\left((1+\lambda)-\frac{2 \lambda}{\theta^{p}} x_{i}^{p}\right)
\end{aligned}
$$

Therefore, the log-likelihood function is given by

$$
\begin{aligned}
\ln \ell & =\ln \left(\prod_{i=1}^{n} p \frac{x_{i}^{p-1}}{\theta^{p}}\left((1+\lambda)-\frac{2 \lambda}{\theta^{p}} x_{i}^{p}\right)\right) \\
& =n \ln p+(p-1) \sum_{i=1}^{n} \ln x_{i}-n p \ln \theta+\sum_{i=1}^{n} \ln \left(1+\lambda-\lambda \frac{x_{i}^{p}}{\theta^{p}}\right)
\end{aligned}
$$

Deriving Equation (20) withe respect to the parameters we get:

$$
\begin{array}{llc}
\frac{\partial \ell}{\partial \theta} & = & \frac{1}{\theta}\left(-n p+\sum_{i=1}^{n} \frac{\lambda x_{i}^{p}}{(1+\lambda) \theta^{p}-\lambda x_{i}^{p}}\right) \\
\frac{\partial \ell}{\partial p}= & \frac{n}{p}+\sum_{i=1}^{n} \ln \left(x_{i}\right)-n \ln (\theta)-\sum_{i=1}^{n} \frac{x_{i}^{p} \ln \left(\frac{x_{i}}{\theta}\right)}{(1+\lambda) \theta^{p}-\lambda x_{i}^{p}} \\
\frac{\partial \ell}{\partial \lambda}= & \sum_{i=1}^{n} \frac{\theta^{p}-x_{i}^{p}}{\theta^{p}-\lambda\left(\theta^{p}-x_{i}^{p}\right)}
\end{array}
$$

Equating the system of derivatives in Equation (21) to zero, we get the following system of equations

$$
\begin{aligned}
\sum_{i=1}^{n} \frac{\lambda x_{i}^{p}}{(1+\lambda) \theta^{p}-\lambda x_{i}^{p}} & =n p \\
\frac{n}{p}-\sum_{i=1}^{n} \frac{x_{i}^{p} \ln \left(\frac{x_{i}}{\theta}\right)}{(1+\lambda) \theta^{p}-\lambda x_{i}^{p}} & =n \ln (\theta)-\sum_{i=1}^{n} \ln \left(x_{i}\right) \\
\sum_{i=1}^{n} \frac{\theta^{p}-x_{i}^{p}}{\theta^{p}-\lambda\left(\theta^{p}-x_{i}^{p}\right)} & =0
\end{aligned}
$$

There is no exact solution for this system of equations. So, to get the maximum likelihood estimates for the distribution parameters, we have to solve this system numerically.

\section{Entropy}

\subsection{Renyi Entropy}

Theorem 7.1 The Renyi entropy of order $\beta \geq 0$ is defined is

$$
E_{\beta}=\frac{1}{1-\beta} \log \sum_{i=0}^{n}\left(\begin{array}{c}
\beta \\
i
\end{array}\right)(1+\lambda)^{i}(-2 \lambda)^{\beta-i} \frac{p^{\beta} \theta^{(1-\beta)}}{2 p \beta-p i-\beta+1}
$$




\section{Proof.}

$$
\begin{gathered}
E_{\beta}=\frac{1}{1-\beta} \log \int_{0}^{\theta}(\psi(x))^{\beta} d x \\
=\frac{1}{1-\beta} \log \int_{0}^{\theta}\left(\frac{(1+\lambda) p}{\theta^{p}} x^{p-1}-\frac{2 \lambda p}{\theta^{2 p}} x^{2 p-1}\right)^{\beta} d x \\
=\frac{1}{1-\beta} \log \int_{0}^{\theta} p^{\beta}\left(\frac{(1+\lambda) x^{p-1}}{\theta^{p}}-\frac{2 \lambda x^{2 p-1}}{\theta^{2 p}}\right)^{\beta} d x \\
=\frac{1}{1-\beta} \log \int_{0}^{\theta} \frac{p^{\beta} x^{\beta(p-1)}}{\theta^{\beta p}}\left((1+\lambda)-\frac{2 \lambda x^{p}}{\theta^{p}}\right)^{\beta} d x \\
=\frac{1}{1-\beta} \log \int_{0}^{\theta} \frac{p^{\beta} x^{\beta(p-1)}}{\theta^{\beta p}}\left(\sum_{i=0}^{n}\left(\begin{array}{l}
\beta \\
i
\end{array}\right)(1+\lambda)^{i}(-2 \lambda)^{\beta-i}\left(\frac{x^{p}}{\theta^{p}}\right)^{\beta-i}\right) d x \\
=\frac{1}{1-\beta} \log \sum_{i=0}^{n}\left(\begin{array}{c}
\beta \\
i
\end{array}\right) \frac{(1+\lambda)^{i}(-2 \lambda)^{\beta-i} p^{\beta}}{\theta^{p(2 \beta-i)}} \int_{0}^{\theta} x^{(2 p \beta-\beta-p i)} d x \\
=\frac{1}{1-\beta} \log \sum_{i=0}^{n}\left(\begin{array}{c}
\beta \\
i
\end{array}\right) \frac{(1+\lambda)^{i}(-2 \lambda)^{\beta-i} p^{\beta}}{\theta^{p(2 \beta-i)}} \frac{\theta^{(2 p \beta-\beta-p i+1)}}{2 p \beta-\beta-p i+1} \\
=\frac{1}{1-\beta} \log \sum_{i=0}^{n}\left(\begin{array}{c}
\beta \\
i
\end{array}\right)(1+\lambda)^{i}(-2 \lambda)^{\beta-i} \frac{p^{\beta} \theta^{(1-\beta)}}{2 p \beta-p i-\beta+1}
\end{gathered}
$$

\subsection{Tsallis Entropy}

Tsallis entropy (Tsallis, 1988) for a continuous random variable is defined as follows:

$$
\begin{aligned}
E_{T}(X) & =\frac{1}{q-1}\left(1-\int_{0}^{\theta}(\psi(x))^{q} d x\right), \quad x \geq 0 \\
& =\frac{1}{q-1}\left[1-\sum_{i=0}^{n}\left(\begin{array}{c}
q \\
i
\end{array}\right)(1+\lambda)^{i}(-2 \lambda)^{q-i} \frac{p^{q} \theta^{(1-q)}}{(2 p q-p i-q+1)}\right]
\end{aligned}
$$

\section{Quantile Function}

The quantile value is a value, say $x$, of the random variable, $X$, with $\operatorname{CDF} \Psi(x)$ such that $\Psi(x)=p(X \leq x)=q$, where $0<q<1$. Therefore, the quantile of the TMI distribution is given by

$$
x=\theta \sqrt[p]{\frac{1+\lambda \pm \sqrt{(1+\lambda)^{2}-4 \lambda q}}{2 \lambda}}, \quad \lambda \neq 0
$$

Proof.

$$
\begin{aligned}
\Psi(x) & =q \\
(1+\lambda) \frac{x^{p}}{\theta^{p}}-\lambda \frac{x^{2 p}}{\theta^{2 p}} & =q
\end{aligned}
$$

Assume $y=\frac{x^{p}}{\theta^{p}}$, then

$$
\begin{aligned}
(1+\lambda) y-\lambda y^{2} & =q \\
\lambda y^{2}-(1+\lambda) y+q & =0
\end{aligned}
$$

Using the general formula for quadratic equations, we get

$$
y=\frac{1+\lambda \pm \sqrt{(1+\lambda)^{2}-4 \lambda q}}{2 \lambda}
$$


replacing $y$ by its value $\frac{x^{p}}{\theta^{p}}$, we have

$$
\begin{aligned}
\frac{x^{p}}{\theta^{p}} & =\frac{1+\lambda \pm \sqrt{(1+\lambda)^{2}-4 \lambda q}}{2 \lambda} \\
x^{p} & =\left(\frac{1+\lambda \pm \sqrt{(1+\lambda)^{2}-4 \lambda q}}{2 \lambda}\right) \theta^{p} \\
\therefore x & =\theta \sqrt[p]{\frac{1+\lambda \pm \sqrt{(1+\lambda)^{2}-4 \lambda q}}{2 \lambda}}
\end{aligned}
$$

The median of a continuous random variable $X$ is defined to be the value $m$ such that $\Psi(m)=\frac{1}{2}$. Hence, the median is the quantile value when $q=\frac{1}{2}$. Therefore,

$$
\begin{aligned}
m & =\theta \sqrt[p]{\frac{1+\lambda \pm \sqrt{(1+\lambda)^{2}-2 \lambda}}{2 \lambda}} \\
& =\theta \sqrt[p]{\frac{1+\lambda \pm \sqrt{1+\lambda^{2}}}{2 \lambda}}
\end{aligned}
$$

\section{Conclusion}

In this paper, a generalization of Mukherjee-Islam distribution of failure time is introduced. It is called the transmuted Mukherjee-Islam distribution. We have studied some properties of this distribution, such as: moments, mean, variance, order statistics, maximum likelihood estimates of the distribution parameters. we, also have found the reliability and hazard rate functions, Renyi and Tsallis entropies and the quantile function as well as the median. The mean and the kurtosis decrease as the value of $\lambda$ increases. The shape of the distribution is left skewed always regardless the value of $p$ and $\lambda$.

\section{References}

Al-Omari, A. I., Al-khazaleh, A. M., \& Alzoubi, L. M. (2017). Transmuted janardan distribution: A generalization of the janardan distribution. Journal of Statistics Applications $\mathcal{E}$ Probability, 5(2), 1-11. https://doi.org/10.18576/jsap/060101

Aryal, G. R., \& Tsokos, C. P. (2011). Transmuted weibull distribution: A generalization of the weibull probability distribution. European Journal of Pure $\mathcal{F}$ Applied Mathematics, 4(2), 89-102.

Aryal, G. R., \& Tsokos, C. P. (2013). On the transmuted extreme value distribution with application. Journal of Statistical Applications $\mathcal{F}$ Probability, 2(1), 11-20. https://doi.org/10.12785/jsap/020102

Ashour, S., \& Eltehiwy, M. (2013). Transmuted lomax distribution. American Journal of Applied Mathematics and Statistics, 1(6), 121-127. https://doi.org/10.12691/ajams-1-6-3

Bourguignon, M., Ghosh, I., \& Cordeiro, G. (2016). General results for the transmuted family of distributions and new models. Journal of Probability and Statistics. https://doi.org/10.1155/2016/7208425

Cordeiro, G. M., Ortega, E. M., \& da Cunha, D. C. (2013). The exponentiated generalized class of distributions. Journal of Data Science, 11, 1-27.

David, H., \& Nagaraja, H. (2003). Order Statistics. John Wiley \& sons, Inc., Hoboken, New Jersey, $3^{\text {rd }}$ edition. https://doi.org/10.1002/0471722162

Elbatal, I., Asha, G., \& Raja, A. V. (2014). Transmuted Exponentaited Frêchet Distribution: Properties and Applications. Journal of Statistics Applications E Probability, 3(3), 379-394.

Khan, M. S., \& King, R. (2013). Transmuted modified weibull distribution: A generalization of the modified weibull probability distribution. European Journal of Pure and Applied Mathematics, 6(1), 66-88.

Khazaleh, A. M. (2016). Transmuted Burr type XII distribution: A generalization of the Burr type XII distribution. International Mathematical Forum, 11(12), 547-556. https://doi.org/10.12988/imf.2016.6443

Merovci, F. (2013a). Transmuted lindley distribution. International Journal of Open Prob-lems in Computer Science and Mathematics, 6, 63-72. https://doi.org/10.12816/0006170 
Merovci, F. (2013b). Transmuted rayleigh distribution. Austarian Journal of Statistics, 42(1), 21-31.

Merovci, F., \& Elbatal, I. (2014). Transmuted Lindley-Geometric Distribution and its Applications. Journal of Statistics Applications E Probability, 3(1), 77-91. https://doi.org/10.18576/jsap/030107

Mukheerji, S. P., \& Islam, A. (1983). A finite range distribution of failures times. Naval Research Logistics Quarterly, 30, 487-491. https://doi.org/10.1002/nav.3800300313

Shaw, W. T., \& Buckley, I. R. (2007). The alchemy of probability distributions: Beyond gram-charlier expansions, and a skew-kurtotic-normal distribution from a rank rransmu-tation map. Technical report.

Tsallis, C. (1988). Possible generalization of boltzmann-gibbs statistics. Journal of Statistical Physics, 52, 479-487. https://doi.org/10.1007/BF01016429

Vardhan, R. V., \& Balaswamy, S. (2016). Transmuted new modified weibull distribution. Mathematical Sciences and Applications E-Notes, 4(1), 125-135.

\section{Copyrights}

Copyright for this article is retained by the author(s), with first publication rights granted to the journal.

This is an open-access article distributed under the terms and conditions of the Creative Commons Attribution license (http://creativecommons.org/licenses/by/4.0/). 